\section{(- OPEN ACCESS}

\title{
Meeting update: faecal microbiota transplantation-bench, bedside, courtroom?
}

\author{
Nik Sheng Ding, ${ }^{1}$ Benjamin H Mullish, ${ }^{2}$ John McLaughlin, ${ }^{3,4}$ Ailsa Hart, ${ }^{5}$ \\ Julian R Marchesi ${ }^{6}$
}

For numbered affiliations see end of article.

\section{Correspondence to}

Professor John McLaughlin, Division of Endocrinology, Diabetes and Gastroenterology, School of Medical Sciences, Faculty of Biology, Medicine and Health and Manchester Academic Health Sciences Centre University of Manchester M13 9PL;

john.mclaughlin@manchester. ac.uk

Received 19 September 2016 Accepted 17 October 2016 Published Online First 03 November 2016

\section{CrossMark}

To cite: Ding NS, Mullish BH, McLaughlin J, et al. Frontline Gastroenterology 2018:9:45-48.

\section{INTRODUCTION}

A group of stakeholders met, under the aegis of the British Society of Gastroenterology, to discuss the current landscape of faecal microbiota transplantation (FMT) within the UK and beyond. The meeting covered a wide range of topics, ranging from the practical aspects of establishing an FMT service and regulatory issues relating to its delivery, to research implications and likely future directions.

\section{CLINICAL EVIDENCE TO DATE}

Case report and case series data supportive of the efficacy of FMT as treatment for recurrent/refractory Clostridium difficile infection (CDI) have slowly accumulated over many decades, but randomised trial data supporting its use for this indication were lacking until as recently as $2013 .{ }^{1}$ There are now a growing number of randomised studies/trials that have consistently demonstrated the much greater efficacy of FMT than that of vancomycin in inducing remission from recurrent/refractory CDI; success from a single FMT is quoted at $\geq 80 \%$, and for two FMTs as $\geq 90 \% .{ }^{1}$ FMT appears to be similarly as efficacious for this indication regardless of whether the transplant is delivered via the upper gastrointestinal (GI) ${ }^{1}$ or lower GI tract. ${ }^{2} 3$

Based on this clinical evidence (along with data supporting the cost effectiveness of FMT in comparison with other treatment strategies), ${ }^{4}$ FMT has now been accepted as an appropriate treatment option for recurrent/refractory CDI by the National Institute for Health and Care Excellence, ${ }^{5}$ Public Health England ${ }^{6}$ and European guidelines. ${ }^{7}$

There is increasing recognition that a distinctive pattern of alteration of the structure of the gut microbiota (or 'dysbiosis') appears to characterise a number of conditions, including inflammatory bowel disease and metabolic syndrome. Although it remains largely unclear as to whether these microbiota changes are a cause of these conditions, consequence or incidental, there is much interest as to whether manipulation of the gut microbiota might be a successful novel therapeutic strategy; however, clinical studies in this area are largely lacking at present. ${ }^{8}$ Two recent randomised controlled trials of FMT as a potential treatment for ulcerative colitis ${ }^{910}$ were largely negative, but included microbiota analysis that suggested that only certain stool donors may be effective, and that matching between donor and recipient may be required.

\section{ESTABLISHING A CLINICAL FMT SERVICE}

The first session focused on the practical aspects of setting up a clinical FMT service, with Dr Benjamin Mullish sharing his experience of establishing a service at Imperial College Healthcare NHS Trust. Although primarily started to treat patients with recurrent/refractory CDI within the Trust itself, the paucity of similar services within the area means that it has ended up as the primary centre for this service within North London.

There are very few exclusion criteria for recipients of FMT within this service; in particular, it was discussed that the currently available clinical evidence for immunosuppressed patients receiving FMT for recurrent CDI has shown a comparable efficacy and limited adverse events. ${ }^{11}$ Imperial have adopted upper GI administration of FMT of stool that has been previously processed and frozen in 
glycerol as cryopreservative, an approach that has been shown to have similar efficacy to transplant freshly processed stool. ${ }^{3}{ }^{12}$ Stool donors are all volunteers, and typically workers within the Trust; a summary of eligibility criteria for acceptance as a donor is listed within box 1 . The discussion focused on the potential practical difficulties in running a service, including establishing and maintaining a screened donor pool, and the ongoing uncertainty regarding the regulation and governance of FMT within the UK. Further discussion included the considerable costs involved in maintaining a service, making it likely to be unfeasible for all hospitals to offer FMT; only about 10-15 centres within the UK appear to offer a formalised FMT service at present. Longer term, a more pragmatic solution for the UK might be establishment of 'FMT networks', with a 'hub' that oversees donor management and transplant preparation, and that treats patients from a number of regional 'spoke' centres.

\section{UNDERSTANDING THE MECHANISMS OF FMT}

The second session was presented by Dr Emma Allen-Vercoe, a microbial ecologist based at the University of Guelph, Canada. Dr Allen-Vercoe discussed that while there has been a recent explosion in literature exploring the changes in the structure of the gut microbiota in different conditions, there has to date been more limited work examining the alteration in the function of the gut microbiota and the interaction between the gut microbiota and the physiology of the host. Interactions between the gut microbiota and host immunology and metabolism have started to help explain the efficacy of FMT for CDI, and may have wider implications as manipulation of the microbiota is explored in a range of conditions. Concepts such as functional redundancy and disability were also introduced. 'Functional redundancy' refers to the pool of gut microorganisms which may absorb any potential insults and be replaced by others with little to no effect on the host, and with no loss of gut microbiota

Box 1 Key criteria used to evaluate potential stool donors to a faecal microbiota transplantation programme:

- $<50$ years old, $\mathrm{BMI}<30$, non-smokers, no alcohol in $<24$ hours, no antibiotic use within 3 months.

- Health questionnaires-personal, family and travel history taken to assess eligibility.

- Screening of blood (viral hepatitis, HIV, etc) and stool for pathogens. Screening programme under ongoing review to ensure suitability and relevance; for example, recent introduction of screening for gut colonisation of multiresistant organisms, including Carbapenem-resistant enterobacteriaceae (CRE). function. In contrast, 'functional disability' describes a microbial ecosystem with little ability to cope with any external insult; in humans, this may have been derived from multiple aetiologies, such as microbiota depletion as a result of repeated antibiotic use (the so-called 'missing microbiota' hypothesis). ${ }^{13}$

\section{ROLE FOR FMT IN NON-GASTROINTESTINAL DISORDERS}

Professor Max Nieuwdorp, an endocrinologist based at the Academic Medical Centre in Amsterdam-and coauthor on the key first randomised trial for FMT in treating CDI-presented his work on the potential role of the gut microbiota in metabolic syndrome. Work in rodents and now in humans has suggested that FMT from lean donors may increase insulin sensitivity in those with metabolic syndrome, and that the delivery of butyrate-producing bacteria may be the key mechanism underlying this. Butyrate-producing strains of bacteria may exert metabolic benefits via a number of routes, including enhancement of mitochondrial activity, suppression of metabolic endotoxaemia and/or activation of intestinal gluconeogenesis. ${ }^{14}$

Professor Claudia Mauri (from University College London) discussed the possible implications of the gut microbiota to rheumatology. IL-1-receptor antagonist knockout mice raised in a germ-free (ie, bacteria-free) environment have no joint disease, but rapidly develop severe $\mathrm{T}$ cell arthritis if raised in conventional housing, or even after exposure to Lactobacillus bifidus only. ${ }^{15}$ Dysbiosis has been described in patients with rheumatoid arthritis, with Porphyromonas gingivalis detected in higher amounts in oral mucosa. ${ }^{15}$ P. gingivalis may have a key mechanistic role in the onset of rheumatoid arthritis, as it activates enzymes which may be responsible for protein citrullination; $30 \%$ of those positive for anticitrullinated peptide antibodies will develop rheumatoid arthritis if found to be positive on screening.

Professor Paul O'Toole-a microbiologist from University College Cork-discussed the contribution of the microbiota to human ageing. His research (the Eldermet project) has shown very distinctive patterns of microbiota alteration among elderly people in different environments (for instance, between people in long-term residential care compared with those living in the community). However, difficulties exist in designing and interpreting studies in this area-for instance, is it the person's living environment that explains the structure of their microbiota per se, or is this just a proxy for their diet, medical comorbidities, etc?

The next topic of discussion was regarding the brain-gut microbiota axis, a long-standing interest of Professor John Cryan and his team at University College Cork. It is increasingly being recognised that there may be a link between the gut microbiota and a range of conditions with a neurological component, 
including hepatic encephalopathy, multiple sclerosis, affective disorders and even irritable bowel syndrome. In rodents, administration of oral non-absorbable antibiotics or FMT produced consistent changes in behaviour and alterations in the expression of brain-derived neurotropic factor. ${ }^{16}$ Similar results have started to emerge for the manipulation of the microbiota in rodent models of neurological and psychiatric disease.

\section{THE FUTURE OF FMT}

Despite the efficacy of FMT for recurrent CDI, it evidently has drawbacks, including its unpalatability, the invasive means of administration and concerns regarding transmission of infection. While oral, capsulised FMT has started to emerge, ${ }^{17}$ this still represents a considerable pill burden in its current form and remains an essentially untargeted therapy. Of particular interest, a group from Canada has recently demonstrated that a 'stool substitute' of 33 pure bacterial strains of commensals isolated from the stool of a healthy person may have similar efficacy in treating CDI as a full FMT. ${ }^{18}$ As the mechanisms underlying the efficacy of FMT continue to emerge, future directions may include yet further refinement, perhaps even to a scenario where bacterial derived products (eg, enzymes) or metabolites alone might replace FMT.

\section{REGULATORY AND GOVERNANCE FRAMEWORK FOR FMT}

FMT providers have voiced concerns regarding ongoing uncertainties regarding the regulation and 'quality assurance' of FMT services in the UK and beyond. Public interest in FMT is high, and already there has started to be the emergence of unregulated private FMT clinics, along with reports of home preparation and administration, with the inherent risks associated with it. Both the British Society of Gastroenterology and Healthcare Infection Society have expressed interest in forming a joint regulatory committee, but none exists at present. Public Health England have sought to engage with FMT providers to define standards of good manufacturing practice/ appropriate governance of an FMT service, although these have not been formalised at present. With a move in North America towards FMT increasingly being provided by commercial 'stool banks' (such as OpenBiome)-and interest from a number of parties in this developing within the UK-such standards will be of key benefit.

Regulation of FMT within the UK has been explored by the Human Tissue Authority (HTA) and the Medicines and Healthcare products Regulatory Agency (MHRA). The HTA have sought clarification from the European Commission about whether FMT would require regulation under the EU Tissues and Cells Directives; the conclusion was that it would not, and that FMT therefore fell outside of the HTA's remit. The MHRA are currently having an ongoing dialogue with FMT providers within the UK about whether FMT is defined as a 'medicinal product' or not, and whether separate regulation for FMT as treatment for CDI and for use in other conditions/within clinical trials may be more appropriate.

\section{CONCLUSIONS}

The huge success of FMT in treatment for CDI has provided a launch pad for interest in the role of the gut microbiota in a range of human diseases, GI and otherwise. Scientifically, the key immediate challenge is to better understand the function of the gut microbiota, and the interaction between the gut microbiota and the host's physiology. Clinically, once discussions regarding regulation have been resolved, refinement of FMT to a more targeted therapy will be a critical next step.

\section{Author affiliations}

${ }^{1}$ Faculty of Medicine, Department of Surgery and Cancer, Imperial College London, London, UK ${ }^{2}$ Division of Digestive Diseases, Department of Surgery and Cancer, St Mary's Hospital Campus, Imperial College London, London, UK

${ }^{3}$ Division of Endocrinology, Diabetes and Gastroenterology, School of Medical Sciences, Faculty of Biology, Medicine and Health, University of Manchester

${ }^{4}$ Salford Royal NHS Foundation Trust and Manchester Academic Health Sciences Centre ${ }^{5}$ Faculty of Medicine, Department of Surgery and Cancer, Northwick Park and St Marks Site, Imperial College London, London, UK

${ }^{6}$ Division of Organisms and Environment, School of Biosciences, Cardiff University, Cardiff, UK

Acknowledgements To Julie Solomon for great talent in organising the meeting on behalf of the BSG.

Competing interests None declared.

Provenance and peer review Not commissioned; internally peer reviewed.

Speakers and topics

Clinical experience of setting up a faecal microbiota transplantation (FMT) service-Benjamin Mullish, Imperial College London

FMT for GI disease (Clostridium difficile infection and IBD)Emma Allen-Vercoe, Univ Guelph, Canada

The potential of FMT for metabolic syndrome-Max Nieuwdorp, Academic Medical Center, Amsterdam

The potential of FMT for rheumatology-Claudia Mauri, University College London

Microbiota of the ageing subject-changes, health associations and modulation prospects-Paul O'Toole, Cork

RePOOPulate: the pros and cons of a synthetic gut microbiota for FMT-Emma Allen-Vercoe, University of Guelph, Canada

FMT and the gut/brain axis-Siobhain O’Mahony, Cork

FMT and the current UK regulatory framework-Victoria McCune and Peter Hawkey, Public Health England Birmingham 
Open Access This is an Open Access article distributed in accordance with the Creative Commons Attribution Non Commercial (CC BY-NC 4.0) license, which permits others to distribute, remix, adapt, build upon this work noncommercially, and license their derivative works on different terms, provided the original work is properly cited and the use is non-commercial. See: http://creativecommons.org/licenses/by$\mathrm{nc} / 4.0 /$

\section{REFERENCES}

1 van Nood E, Vrieze A, Nieuwdorp M, et al. Duodenal infusion of donor feces for recurrent clostridium difficile. $N$ Engl J Med 2013;368:407-15.

2 Cammarota G, Masucci L, Ianiro G, et al. Randomised clinical trial: faecal microbiota transplantation by colonoscopy vs. vancomycin for the treatment of recurrent Clostridium difficile infection. Aliment Pharmacol Ther 2015:41;835-43.

3 Youngster I, Sauk J, Pindar C, et al. Fecal microbiota transplant for relapsing Clostridium difficile infection using a frozen inoculum from unrelated donors: a randomized, open-label, controlled pilot study. Clin Infect Dis 2014;58:1515-22.

4 Konijeti GG, Sauk J, Shrime MG, et al. Cost-effectiveness of competing strategies for management of recurrent Clostridium difficile infection: a decision analysis. Clin Infect Dis 2014;58:1507-14.

5 Faecal microbiota transplant for recurrent Clostridium difficile infection. Interventional procedures guidance [IPG485]. Published: March 2014 https://www.nice.org.uk/guidance/ ipg485 (accessed 30 Sep 2016).

6 Updated guidance on the management and treatment of Clostridium difficile infection. Public Health England. Published: May 2013. https://www.gov.uk/government/uploads/ system/uploads/attachment_data/file/321891/Clostridium difficile_management_and_treatment.pdf (accessed 30 Sep 2016).

7 Debast SB, Bauer MP, Kuijper EG, et al. European Society of Clinical Microbiology and Infectious diseases: update of the treatment guidance document for Clostridium difficile infection. Clin Microbiol Infect 2014;20(Suppl 2):1-26.

8 Smits LP, Bouter K, de Vos WM, et al. Therapeutic potential of fecal microbiota transplantation. Gastroenterology 2013;145:946-53.

9 Moayyedi P, Surette MG, Kim PT, et al. Fecal microbiota transplantation induces remission in patients with active ulcerative colitis in a randomized controlled trial. Gastroenterology 2015;149:102-9.

10 Rossen NG, Fuentes S, van der Spek MJ, et al. Findings from a randomized controlled trial of fecal transplantation for patients with ulcerative colitis. Gastroenterology 2015;149:110-18.

11 Kelly CR, Ihunnah C, Fischer M, et al. Fecal microbiota transplant for treatment of Clostridium difficile infection in immunocompromised patients. Am J Gastroenterol 2014;109:1065-71.

12 Hamilton MJ, Weingarden AR, Sadowsky MJ, et al. Standardized frozen preparation for transplantation of fecal microbiota for recurrent Clostridium difficile infection. Am J Gastroenterol 2012;107:761-7.

13 Blaser MJ, Falkow S. What are the consequences of the disappearing human microbiota? Nat Rev Microbiol 2009;7:887-94.

14 Hartstra AV, Bouter KEC, Bäckhed F, et al. Insights into the role of the microbiome in obesity and type 2 diabetes. Diabetes Care 2015;38:159-65.

15 Scher JU, Abramson SB. The microbiome and rheumatoid arthritis. Nat Rev Rheumatol 2011;7:569-78.

16 Bercik P, Denou E, Collins J, et al. The intestinal microbiota affect central levels of brain-derived neurotropic factor and behavior in mice. Gastroenterology 2011;141:599-609, 609.e1-3.

17 Youngster I, Russell GH, Pindar C, et al. Oral, capsulized, frozen fecal microbiota transplantation for relapsing Clostridium difficile infection. JAMA 2014;312:1772-8.

18 Petrof EO, Gloor GB, Vanner SJ, et al. Stool substitute transplant therapy for the eradication of Clostridium difficile infection: 'RePOOPulating' the gut. Microbiome 2013;1:3. 